Musées, Patrimoine et Culture scientifiques et techniques

$153 \mid 2014$

mai-juin 2014

\title{
Les lots importants d'objets de petite taille
}

Muriel Lecouvez

URL : http://journals.openedition.org/ocim/1377

DOI : $10.4000 /$ ocim. 1377

ISSN : 2108-646X

Éditeur

OCIM

Édition imprimée

Date de publication : 25 juin 2014

ISSN : 0994-1908

\section{Référence électronique}

Muriel Lecouvez, «Les lots importants d'objets de petite taille », La Lettre de I'OCIM [En ligne], 153 | 2014, mis en ligne le 25 juin 2016, consulté le 30 avril 2019. URL : http:// journals.openedition.org/ocim/1377; DOI : 10.4000/ocim.1377 


\title{
Les lots importants d'objets de petite taille
}

\author{
Muriel Lecouvez \\ Attachée de conservation du Patrimoine (PSTN), \\ responsable des collections de zoologie \\ au musée d'Histoire naturelle de Lille
}

Le musée d'Histoire naturelle de Lille conserve un ensemble d'environ 460000 objets, répartis dans quatre départements : zoologie (205 000), géologie (180 000), sciences et techniques $(60000)$ et ethnographie (13 000). Lors des opérations de récolement, le traitement de collections numériquement très importantes d'objets de petite taille implique la mise en place de stratégies particulières. Sont surtout concernées les collections de sciences naturelles : insectes, mollusques, pétrographie, paléontologie, archéologie.

La taille limite des éléments est de l'ordre de 2 à 3 millimètres, taille minimale pour qu'ils soient identifiés et comptabilisés. En dessous, il n'y a pas de dénombrement possible et on considère qu'il s'agit d'un élément et non d'un lot. En pétrographie (collection de roches) par exemple, l'élément est la roche, qu'elle soit fragmentaire ou non : un sable, un argile, un grès... Dans ce cas, le nombre d'objets est égal à 1 et la quantification se fait par une mesure du poids de l'échantillon.

Tout au long du récolement décennal peuvent être combinées des opérations de récolement détaillé et de récolement « global » plus rapide afin de pouvoir traiter l'ensemble des collections. Lors de l'inventaire déjà, les

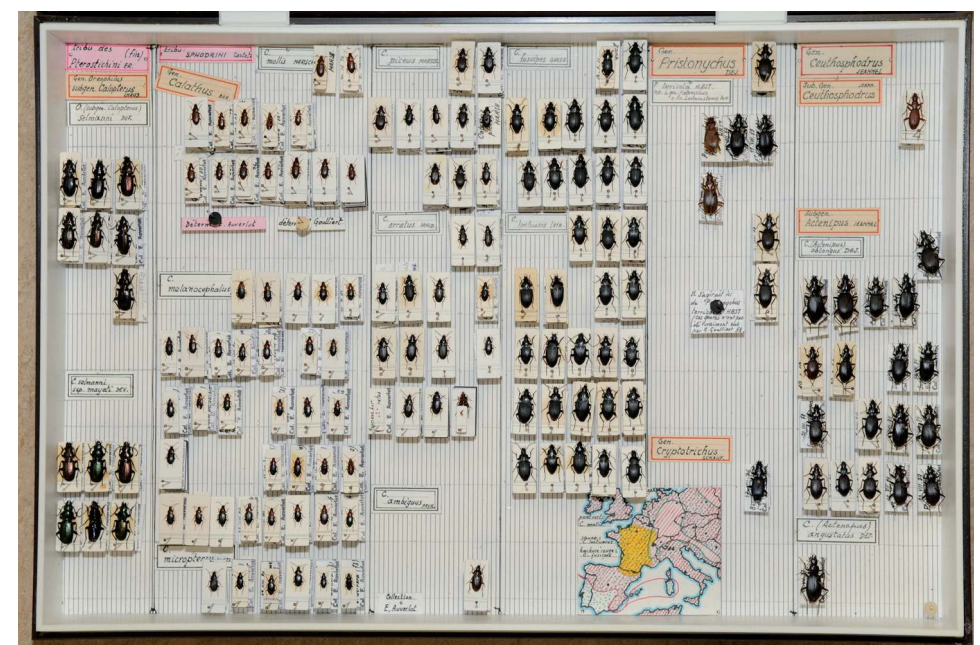

Boîte entomologique contenant 165 spécimens divisés en 15 groupes comprenant de 1 à 31 spécimens. c) Musée d'Histoire naturelle de Lille objets de petite taille sont souvent traités par lot dès leur enregistrement. Les lots d'objets de petite taille sont d'autant plus importants numériquement qu'ils correspondent à des lots complexes : boîtes de petits fossiles, de coquillages ou d'insectes qui contiennent plusieurs espèces mélangées. À partir des groupes identifiables, le lot est découpé en sous-parties au sein desquelles chaque élément est compté, par exemple :

- une boîte entomologique contenant 165 spécimens sera divisée en 15 groupes de 1 à 31 spécimens ;

- une boîte de coquillages rassemble 704 spécimens répartis dans 54 casiers de 4 à 273 spécimens.

La partition facilite le comptage des éléments et permet de réaliser rapidement un pointage des contenants lors du récolement. Elle peut s'appuyer sur les informations scientifiques disponibles mais peut aussi être effectuée par des non-spécialistes à partir de critères de ressemblance, l'objectif de l'opération n'étant pas d'étudier la collection mais plutôt d'en faciliter la gestion. Le travail d'inventaire spécifique sera alors organisé dans un deuxième temps, avec l'aide de spécialistes, en fonction des groupes identifiés.

Si tous les éléments ne sont pas identifiables facilement, il est aussi possible d'enregistrer le lot complexe dans son ensemble, et seuls les éléments les plus intéressants, du point de vue scientifique ou muséographique, sont inventoriés individuellement.

Dans le protocole de saisie, il est nécessaire de définir clairement la limite à partir de laquelle les éléments ne sont plus comptés afin d'éviter des disparités (un lot d'une cinquantaine de coquillages noté «nombreux » d'un coté et une boîte de plus de 100 spécimens comptés précisément de l'autre). Si la limite est trop basse, le risque est de regrouper dans une catégorie «nombreux » des quantités très différentes (50 à 500 par exemple). Lutilisation d'un chiffre rond (1 000/10 000) pour coder des impressions de quantités s'avère peu efficace car trop subjectif. De plus ces « faux » chiffres vont fortement modifier la somme totale de spécimens.

L'expérience montre quau-delà de la centaine, il est fastidieux de compter les éléments et le risque d'erreur augmente rapidement. Il faut donc garder à l'esprit qu'il y 


\section{Points de vue complémentaires}

a une certaine marge d'erreur autour de ces chiffres et que le re-comptage ne suffit pas à garantir la présence de tous les spécimens lors du récolement. Toutefois, ces chiffres peuvent être additionnés et donnent une meilleure idée de la quantité d'objets en collection que les indications «nombreux » sur les fiches d'inventaire. Autrement dit, ces comptages donnent une idée des quantités à traiter et aident à l'organisation du travail, mais naugmentent pas la connaissance des collections et ne protègent pas contre les disparitions.

Les lots importants d'objets de petites tailles peuvent peser considérablement sur le temps de travail si l'on cherche à réaliser un pointage exhaustif. Dans l'objectif d'un inventaire/récolement rapide, il est plus judicieux de se concentrer sur l'identification des contenants avec une photographie et une indication de quantité (dénombrement des sous-parties ou poids), et de réaliser le travail scientifique indépendamment de ces opérations de gestion des collections.

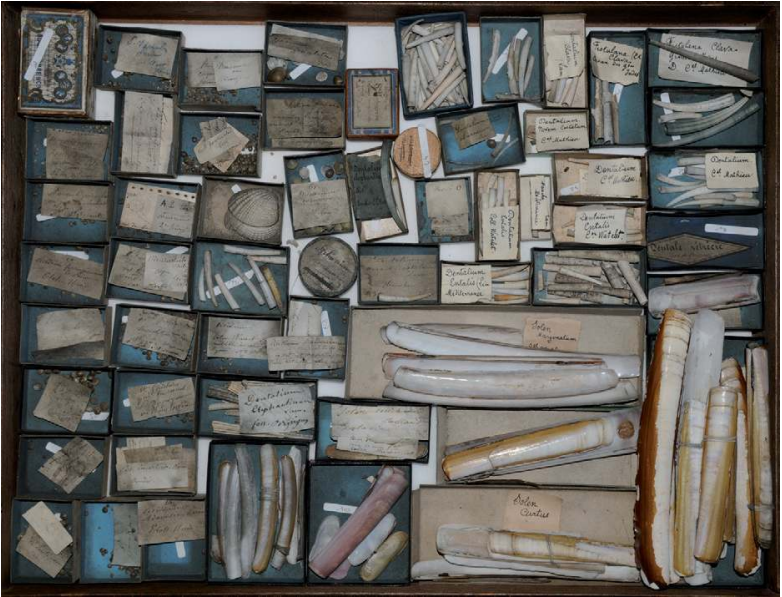

Boîte de coquillages rassemblant 704 spécimens répartis dans 54 casiers comprenant de 4 à 273 spécimens. (c) Musée d'Histoire naturelle de Lille 